\title{
COMPETENCIAS EM DESENVOLVIMENTO DE PRODUTOS E GERENCIAMENTO DE PROJETOS: UM ESTUDO SOBRE AS EMPRESAS JUNIORES DA UNIVERSIDADE FEDERAL DE UBERLÂNDIA
}

Livia Ferreira Seyfried (liviaseyfried@gmail.com) - Faculdade de Gestão e Negócios, Universidade Federal de Uberlândia

Camila de Araujo (camiladearaujo@ufu.br) - Faculdade de Gestão e Negócios, Universidade Federal de Uberlândia

\section{RESUMO}

No contexto universitário as empresas juniores (EJs) representam o ambiente de integração com o mercado profissional, onde os alunos podem colocar em prática o conteúdo acadêmico. $\mathrm{Na}$ Universidade Federal de Uberlândia (UFU) existem mais de 20 EJs, que atuam em diversas áreas. Como os produtos oferecidos pelas empresas são desenvolvidos por projetos, de acordo com a especialidade da cada empresa júnior, as competências em Processo de Desenvolvimento de Produto $(P D P)$ e em Gerenciamento de Projetos (GP) mostram-se primordiais para o sucesso de seus produtos, bem como para a qualificação profissional de seus participantes. Questiona-se, então, se as EJs da UFU possuem tais competências e como elas são aplicadas. Assim, o presente estudo tem como objetivo principal apresentar o cenário das competências em Processo de Desenvolvimento de Produtos e em Gerenciamento de Projetos das empresas juniores da Universidade Federal de Uberlândia. Para o desenvolvimento de tal objetivo foi realizado um estudo de caso múltiplo nas EJS da UFU. Também foram analisadas as disciplinas curriculares de cada curso de graduação das EJs, para verificar se são oferecidos os conteúdos teórico-práticos sobre PDP e GP. As informações de cada empresa foram analisadas e um cenário das EJs da UFU foi montado a partir dos casos estudados. O cenário mostra que as EJs da UFU ainda necessitam do desenvolvimento de competências tanto em Processo de Desenvolvimento de Produto e quanto em Gerenciamento de Projetos. Também são apresentadas propostas de melhoria e capacitação das EJs nas competências que são foco deste estudo. Acredita-se que a partir do resultado deste trabalho possam ser desenvolvidos espaços mais qualificados de aprendizagem profissional, bem como estimular a própria universidade a acompanhar suas EJs com ações efetivas, seja para a atualização curricular de seus cursos, seja com desenvolvimento de ações de capacitação, de forma a gerar uma maior extensão com as organizações que possam ser atendidas pelas EJs.

Palavras chave: Empresa Júnior; Competências; Processo de Desenvolvimento de Produtos; Gerenciamento de Projetos

Área: Educação para desenvolvimento de produtos e serviços

\section{INTRODUÇÃO}

No contexto universitário as empresas juniores (EJs) representam o ambiente de integração com o mercado profissional, onde os alunos podem colocar em prática o conteúdo acadêmico proveniente da sala de aula. Atualmente na Universidade Federal de Uberlândia (UFU) existe o Núcleo de Empresas Juniores (NEJ), que tem por finalidade realizar o intercâmbio de 
informações entre as EJs vinculadas à universidade. São vinte e uma (21) empresas associadas ao NEJ, atuantes em diversas áreas de conhecimento.

Como os produtos oferecidos pelas empresas são desenvolvidos por projetos, em sua maioria no formato de consultoria, de acordo com a especialidade da cada empresa júnior, as competências em Processo de Desenvolvimento de Produto (PDP) e em Gerenciamento de Projetos (GP) mostram-se primordiais para o sucesso de seus produtos, bem como para a qualificação profissional de seus participantes. Questiona-se, então, se as EJs da UFU possuem tais competências e como elas são aplicadas. Assim, o presente estudo tem como objetivo principal apresentar o cenário das competências em Processo de Desenvolvimento de Produtos e em Gerenciamento de Projetos das empresas juniores da Universidade Federal de Uberlândia.

Este trabalho está estruturado em quatro seções apresentadas a seguir: Embasamento teórico, onde são apresentados os principais conceitos sobre empresa júnior, gerenciamento de projetos e desenvolvimento de produtos; Aspectos metodológicos, apresentando como a pesquisa foi realizada; Estudos de casos, onde é apresentada a caracterização das empresas estudadas, os resultados, a análise e, por fim a seção de conclusão com as propostas.

\section{EMBASAMENTO TEÓRICO}

\subsection{Empresa júnior}

Uma EJ é uma organização, sem fins lucrativos, constituída pelo agrupamento de alunos que estejam inscritos em ao menos um curso de graduação vinculado à uma Instituição de Ensino Superior (IES), pública ou privada. São conduzidas de maneira integral por alunos, isto é, tanto as atividades de gestão organizacional como as atividades de prestação de serviços e projetos. Possuem como objetivo principal auxiliar a melhor preparação dos estudantes para o mercado de trabalho, instituindo relação entre a teorias e a prática (BRASIL JUNIOR, 2003). As EJs possuem apoio físico e de infraestrutura das IES para que possam efetuar o desenvolvimento dos trabalhos, porém são regidas por estatutos internos, além de possuir estrutura jurídica própria (BRASIL JUNIOR, 2003; MORETTO NETO et al., 2004).

O conceito de Empresa Júnior (EJ) surgiu em 1967 através da elaboração do projeto de Bernard Caioso da ESSEC (L'Ecole Supérieure dês Sciences Economiques et Commerciales de Paris), com o objetivo de disponibilizar trabalhos administrativos com preços mais acessíveis do que os das empresas tradicionais. Assim, as EJs se disseminaram para diversos cursos e instituições superiores (MORETTO NETO et al., 2004). Em 1986 existiam mais de 100 EJs na França. Assim, em 1990, foi criada a Junior Association for Development in Europe (JADE), sendo essa a confederação europeia de EJs (DIAS; OLIVEIRA; MARCELINO, 2017).

O conceito de EJ chegou ao Brasil em 1987 com o intermédio da Câmara de Comércio França-Brasil na forma de um convite de participação, o qual foi aceito pela Fundação Getúlio Vargas (FGV), pela Fundação Armando Álvares Penteado (FAAP) e pela Escola Politécnica da Universidade de São Paulo. Em 1988 o curso de administração de FGV deu início às suas atividades na EJ, executando serviços de assessoria empresarial em diversas áreas administrativas (DIAS; OLIVEIRA; MARCELINO, 2017; MATOS, 1997). Em 2016 foi sancionada a lei 13.267, que regulamenta as EJs no Brasil (BRASIL JUNIOR, 2016a).

Os principais clientes das EJs são micro e pequenas empresas, assim como organizações do terceiro setor (BRASIL JUNIOR, 2003), que recorrem a alternativas de custo mais baixo, porém qualificados para que possam solucionar os seus problemas. Tal combinação caracteriza benefício para ambos os lados, já que por um lado proporciona um serviço de 
qualidade com baixo custo para as empresas clientes e, por outro lado, conhecimento e experiência para os membros da EJ. Com tais benefícios, o mercado de consultoria de EJs se mantém aquecido, instigando novas práticas com os conceitos aprendidos na teoria, sendo tal ambiente promovido pelo cenário mais informal devido a burocracia mais simplificada das EJs (DIAS; OLIVEIRA; MARCELINO, 2017).

Conforme dados da Confederação Brasileira de Empresas Juniores (BRASIL JUNIOR, 2016b), em 2015 existiam mais de 1200 organizações no Brasil e, somente no ano de 2015, foram executados aproximadamente 2700 projetos e consultorias com a participação de mais de 11.000 universitários, movimentando $\mathrm{R} \$ 10$ milhões na economia brasileira. Em 2016, obteve-se uma receita de $\mathrm{R} \$ 11,1$ milhões, além de 4900 projetos comerciais, com a participação de mais de 15.000 empresários juniores, comprovando a expansão no mercado de EJs no país.

O cenário expansivo, informal e preparatório para o mercado de trabalho mostra-se positivo para o desenvolvimento de alunos de cursos de graduação, por possibilitar a execução de trabalhos com sentido prático e o desenvolvimento de competências, assim como a prática dos julgamentos e livre-arbítrio, para o conhecimento da evolução dos seus desempenhos e ajuste desses (DIAS; OLIVEIRA; MARCELINO, 2017).

\subsection{Gerenciamento de projetos}

Um projeto é considerado um esforço temporário utilizado para a criação de um produto e/ou um serviço único ou então um resultado exclusivo, portando características de início e fim definidos, além de uma elaboração progressiva, pois são efetuadas realizações de maneira incremental (PMI, 2013). Weis e Wysocki (1992) estabelecem que um projeto possui características de complexidade, possuindo recursos limitados com envolvimento interfuncional além de escalonamento de tarefas orientados por objetivos para obter um produto/serviço final.

Uma vez que o gerenciamento de um projeto é composto por dez áreas do conhecimento: integração, escopo, tempo, custo, qualidade, recursos humanos, comunicação, aquisição, riscos e envolvidos (stakeholders) (PMI, 2013), os projetos não devem ser vistos de maneira isolada em uma única área das organizações, mas estão envolvidos em um contexto social (GRABHER, 2002). Considerando tal contexto, autores como Rabechini Junior et al (2011) tem tratado a compreensão do gerenciamento de projetos nas organizações relacionada a três tipos de competências: 1) as individuais, como aptidões e habilidades de cada indivíduo a respeito da solução de problemas; 2) as competências da equipe, relacionadas na capacidade multidisciplinar de resolução de problemas complexos; e 3) as competências da empresa, sendo a capacidade na criação de ambiente capaz de gerar um maior envolvimento do indivíduo e da equipe para que possam executar os projetos eficazmente.

A estrutura organizacional adotada pelas empresas influencia diretamente no âmbito das competências de GP. Assim, em ambientes inovadores, onde as empresas necessitam do envolvimento de diversas áreas funcionais, com a necessidade da diversificação de competências, assim como integração das tecnologias envolvidas no desenvolvimento de novos produtos/serviços e processos, bem como a necessidade de integração para resolução de problemas, a utilização de estrutura funcional acaba limitando a realização dos projetos. Dessa forma, as organizações buscam formas organizacionais diferenciadas da estrutura funcional (PATAH; CARVALHO, 2009).

Vieira et al. (2003) identificaram na EJ da FEA-USP que a estrutura organizacional mais efetiva para eles é o modelo de estrutura por projetos, já que efetuavam projetos com frequência de curta duração. Tal estudo vai ao encontro do Conceito Nacional de Empresa 
Junior (CNEJ), que estabelece que uma EJ deve realizar seu trabalho por meio de projetos e serviços que contribuam para a formação de profissionais capacitados e comprometidos (BRASIL JUNIOR, 2003). Tais dados demonstram que a utilização do gerenciamento de projetos se torna importante para as EJs.

\subsection{Desenvolvimento de produto}

A transformação de dados em oportunidades de mercado, assim como possibilidades técnicas em bens além de informações para a fabricação de um produto comercial, fazem parte do processo de desenvolvimento de produtos (CLARK; FUJIMOTO, 1991). O processo de desenvolvimento de produtos é uma atividade estratégica que busca detectar os desejos e as necessidades que são requisitadas pelo consumidor, entregando então produtos inovadores, projetados de maneira otimizada, além de serem bem executados (RABECHINI JUNIOR et al, 2002). Mas o conceito de PDP vai além de considerar os desejos dos clientes, envolvendo as estratégias competitivas e de produto da empresa, sendo composto por um conjunto de atividades que visa gerar valor para o cliente (PAHL et al., 2007; ROZENFELD et al., 2006; ULRICH; EPPINGER, 2012).

O desenvolvimento de produtos se associa com inúmeras funções de uma empresa, tornandose assim, um dos processos mais complexos de uma organização, com características multidisciplinar, contando com informações e habilidades dos colaboradores de todas as áreas funcionais, além de cada projeto de produto apresentar particularidades e históricos específicos (MUNDIM et al., 2002).

Krishnan e Ulrich (2001) afirmam que existem alguns fatores críticos de sucesso para o PDP, como o preço e posicionamento do produto, a identificação e atendimento das necessidades dos clientes, o alinhamento organizacional, as características dos componentes da equipe de desenvolvimento e a otimização do desempenho. Como os processos do projeto de um produto não ocorrem sucessivamente e os mesmos envolvem pessoas de forma consecutiva, sendo necessária a integração dos indivíduos (MUNDIM et al., 2002), a maneira mais adequada de se conservar o conhecimento que será gerado e manter as características/padrões que foram estabelecidos é a utilização de modelos de referência no processo de desenvolvimento de produtos. Isso ocorre também pelo fato de que as pessoas envolvidas em tal processo nem sempre serão as mesmas, então para que se garanta que o processo e o conhecimento compreendido não estejam vinculados às pessoas, mas sim à empresa, é importante a utilização de tais modelos, principalmente no planejamento de desenvolvimento de novos produtos, já que ele envolve diversos setores em momentos distintos, o que o torna sua compreensão mais complexa do que a de outros processos (ROZENFELD et al., 2006).

Considerando tal teoria, então para as EJs o desenvolvimento de modelos de referência para o PDP mostra-se importante, dado a rotatividade de seus membros. Tal adoção permite tanto uma exequibilidade padronizada dos projetos de novos produtos quanto um contato dos membros das EJs com um PDP mais estruturado.

\section{ASPECTOS METODOLÓGICOS}

Considerando que objetivo principal desta pesquisa é apresentar o cenário das competências em Processo de Desenvolvimento de Produtos e em Gerenciamento de Projetos das empresas juniores da Universidade Federal de Uberlândia, adotou-se uma abordagem qualitativa, uma vez que com tal abordagem um fenômeno pode ser melhor compreendido no contexto em que ocorre e do qual é parte, devendo ser analisado numa perspectiva integrada (GODOY, 1995).

Quanto aos procedimentos para tal abordagem foram realizados dois procedimentos-base: levantamento bibliográfico, para o embasamento teórico e o estudo de caso múltiplo, sendo 
esse último de caráter exploratório. A unidade de análise empregada foi a estrutura de cada EJ.

Para a realização dos estudos de caso, primeiramente foram levantadas quantas EJs estavam ligadas à UFU no primeiro semestre de 2017. Tal levantamento foi feito a partir das informações disponíveis na página do NEJ UFU (Núcleo de Empresas Juniores da UFU) em uma rede social (NEJ UFU, 2017). Foi encontrado um total de vinte e uma (21) empresas juniores. A partir dessa informação foi estruturado um questionário com o objetivo de caracterizar os alunos participantes das EJs, bem como levantar as informações se tais alunos possuem alguma formação em GP e PDP, advinda do curso de graduação ou externa. Um roteiro estruturado de entrevista foi elaborado para os diretores das EJs responderem. Esse último foi dividido em três seções, sendo: 1) caracterização da EJ, 2) aplicação dos conhecimentos de GP e 3) aplicação dos conhecimentos de PDP. Para a seção sobre conhecimentos em GP foi utilizado como base o questionário de Rabechini Junior et al. (2011), considerando as três dimensões apresentadas na literatura: indivíduo, equipe e organização, destacando-se que a dimensão da organização considera as áreas de conhecimento do PMBOK (PMI, 2013). Já para a seção sobre conhecimentos de PDP foram utilizadas as atividades apresentadas no modelo de maturidade de Rozenfeld et al. (2006, p. 485).

Todas as EJs foram convidadas a participar da pesquisa por e-mail e telefonemas. Onze (11) empresas aceitaram participaram da pesquisa. O questionário foi disponibilizado de forma online para essas empresas. Do total de duzentos e quarenta e dois (242) alunos integrantes das EJs participantes, oitenta e quatro (84) alunos responderam ao questionário de caracterização.

A partir das respostas dos questionários, os diretores das EJs foram entrevistados utilizando o roteiro estruturado. Após as entrevistas foram relacionados os cursos de graduação envolvidos com as EJs e assim o currículo de cada um desses cursos foi analisado, para verificar se são oferecidos os conteúdos teórico-práticos sobre PDP e GP.

\section{ESTUDOS DE CASOS}

\subsection{Caracterização das empresas estudadas}

Os cursos de graduação relacionados com as onze empresas estudadas são: Administração, Biotecnologia, Ciências Contábeis, Direito, Gestão da Informação, Engenharia Aeronáutica, Engenharia Ambiental, Engenharia de Alimentos, Engenharia Civil, Engenharia Elétrica e de Telecomunicações, Engenharia Química, Engenharia Mecânica, Engenharia Mecatrônica, Engenharia de Produção e Química Industrial.

O tempo de existência das empresas apresentou uma grande variação, sendo a mais nova com existência de somente 6 meses. As três empresas mais velhas contam com mais de vinte (20) anos de existência e estão relacionadas aos cursos mais antigos da UFU: Administração, Engenharia Mecânica e Ciências Contábeis.

Quanto aos produtos oferecidos por cada EJ, esses estão diretamente relacionados com as competências desenvolvidas nos respectivos cursos de graduação e são ofertados na forma de projetos de consultoria. $\mathrm{O}$ quadro 1 apresenta um resumo das onze empresas participantes da pesquisa, com o tempo de existência, o número de integrantes e seus principais produtos.

Uma vez que o trabalho das EJs é realizado por projetos, a estrutura organizacional adotada pelas EJs da UFU é a estrutura matricial forte. Todos seus integrantes participam dos projetos de consultoria prestados e são alocados nas áreas funcionais que demonstram maior afinidade 
após a fase de "trainee", como são denominados os ingressantes. As áreas funcionais apresentadas por todas as empresas são: Presidência, Projetos, Administrativo-Financeiro, Marketing e Gestão de Pessoas.

Quadro 1. Resumo da caracterização das Empresas juniores estudadas. Fonte: autoria própria

\begin{tabular}{|c|c|c|c|c|}
\hline $\begin{array}{c}\text { ID da } \\
\text { Empresa }\end{array}$ & $\begin{array}{l}\text { Cursos e respectivos } \\
\text { institutos/faculdades }\end{array}$ & $\begin{array}{l}\text { Tempo de } \\
\text { Existência }\end{array}$ & $\begin{array}{l}\text { Número de } \\
\text { integrantes }\end{array}$ & Principais produtos \\
\hline 1 & $\begin{array}{l}\text { Administração e Gestão da } \\
\text { Informação (FAGEN) }\end{array}$ & 24 anos & 35 & $\begin{array}{l}\text { Consultoria sobre } \\
\text { Estratégia, Financeiro, } \\
\text { Gestão de Pessoas e } \\
\text { Marketing }\end{array}$ \\
\hline 2 & Administração (FACIP) & $\begin{array}{c}2 \text { anos e } 6 \\
\text { meses }\end{array}$ & 15 & $\begin{array}{c}\text { Consultoria sobre Gestão } \\
\text { Administrativa em geral }\end{array}$ \\
\hline 3 & Ciências Contábeis (FACIC) & 15 anos & 15 & $\begin{array}{l}\text { Auditoria Contábil, } \\
\text { Consultoria Tributária, } \\
\text { Consultoria Gerencial }\end{array}$ \\
\hline 4 & Direito (FADIR) & 1 ano & 15 & $\begin{array}{c}\text { Consultoria na área } \\
\text { jurídica }\end{array}$ \\
\hline 5 & $\begin{array}{c}\text { Engenharia Aeronáutica, } \\
\text { Engenharia Mecânica e Engenharia } \\
\text { Mecatrônica (FEMEC) }\end{array}$ & 24 anos & 35 & $\begin{array}{c}\text { Consultoria sobre projetos } \\
\text { mecânicos, otimização de } \\
\text { maquinário, materiais e } \\
\text { segurança do trabalho }\end{array}$ \\
\hline 6 & Engenharia Ambiental (ICIAG) & 3 anos & 22 & $\begin{array}{l}\text { Consultoria sobre Gestão } \\
\text { de Resíduos Sólidos, } \\
\text { Regularização Ambiental } \\
\text { e Reaproveitamento de } \\
\text { Água e Energia }\end{array}$ \\
\hline 7 & $\begin{array}{c}\text { Biotecnologia, Engenharia de } \\
\text { Alimentos, Engenharia de } \\
\text { Eletrônica e Telecomunicações } \\
\text { (Campus Patos de Minas - INGEB, } \\
\text { FEQ, FEELT) }\end{array}$ & $\begin{array}{l}1 \text { ano e } 6 \\
\text { meses }\end{array}$ & 20 & $\begin{array}{c}\text { Consultoria em } \\
\text { Biotecnologia, Engenharia } \\
\text { de Alimentos e Eletrônica } \\
\text { e telecomunicações }\end{array}$ \\
\hline 8 & Engenharia Civil (FECIV) & 4 anos & 20 & $\begin{array}{c}\text { Estudo de causa, } \\
\text { Levantamento cadastral, } \\
\text { Memorial descritivo } \\
\end{array}$ \\
\hline 9 & $\begin{array}{c}\text { Engenharia Química (FEQ em } \\
\text { Uberlândia) }\end{array}$ & 4 anos & 30 & $\begin{array}{l}\text { Consultoria em Gestão } \\
\text { Ambiental, Processos } \\
\text { Industriais e Segurança } \\
\text { Industrial }\end{array}$ \\
\hline 10 & Engenharia de Produção (FACIP) & 4 anos & 25 & $\begin{array}{c}\text { Consultoria sobre } \\
\text { Qualidade, Planejamento } \\
\text { da Produção e } \\
\text { Planejamento Estratégico } \\
\end{array}$ \\
\hline 11 & Química Industrial (IQ) & 6 meses & 10 & $\begin{array}{c}\text { Em fase de implantação da } \\
\text { empresa e ainda sem } \\
\text { definição dos produtos }\end{array}$ \\
\hline
\end{tabular}




\subsection{Resultados}

Os resultados foram classificados quanto: 1) maturidade em PDP; 2) Dimensão Indivíduo em GP; 3) Dimensão Equipe em GP, 4) Dimensão Organização em GP e 5) Disciplinas curriculares.

No que concerne à maturidade em PDP, de acordo com as respostas obtidas, todas as EJs participantes da pesquisa podem ser classificadas no primeiro nível de maturidade do PDP. As atividades dentro do PDP que recebem mais atenção estão no fim da macrofase de prédesenvolvimento, com elaboração de escopo, definição de atividades macro e estimativa de tempo. As EJs possuem também um planejamento do portfólio, de acordo com as necessidades de mercado que são percebidas. Porém, observa-se um conhecimento insipiente sobre as demais atividades do PDP. Muitas EJs consideram que as atividades de PDP não se aplicam ao tipo de produto que elas oferecem e, mesmo as empresas juniores que desenvolvem projetos de produtos físicos, como por exemplo a EJ relacionada à Engenharia Mecânica, e que poderiam ter uma atuação mais intensa até a fase do projeto detalhado, não realizam atividades características como o desenvolvimento de concepções alternativas.

Já quanto às competências de Gerenciamento de Projetos, na dimensão Indivíduo, os entrevistados julgam baixas as competências dos indivíduos, sem conhecimento das ferramentas e técnicas mais difundidas e não sabem expressar o que deve ser feito para se atingir uma maturidade em GP. Na dimensão Equipe das competências de GP, os resultados são mais positivos e apontam um maior envolvimento de todos, com disseminação dos objetivos do projeto, reuniões de acompanhamento, controle de prazos e de mudanças. Porém as EJs não realizam adequadamente o acompanhamento dos custos, como o Gerenciamento do Valor Agregado. Em relação à dimensão Organização em GP, existe uma grande diferença entre as EJs quanto à definição de um método formalizado para gerenciar seus projetos. Muitas não possuem nenhum método para gerenciar seus projetos. Algumas EJs possuem formalizado um fluxograma das ações que devem ser realizadas para todo projeto novo, isto é, um processo definido, mas não possuem definidas as técnicas e ferramentas de controle e monitoramento para um projeto em andamento além das reuniões periódicas de acompanhamento. Das áreas de conhecimento do PMBOK (PMI, 2013), as EJs estudadas somente realizam o planejamento e controle dos prazos e do escopo. As demais áreas ainda não são consideradas para planejamento e controle. Considerando a abordagem de GP tradicional ou ágil - adotada pelas EJs, pode-se afirmar que as práticas utilizadas são enquadradas na abordagem tradicional.

Por fim, quanto as disciplinas curriculares dos cursos envolvidos nas EJs, somente dois cursos apresentam uma disciplina voltada para o gerenciamento de projetos. Os cursos de Administração e Gestão da Informação em Uberlândia contam uma disciplina obrigatória de Gerenciamento de Projetos em suas grades, com carga horária de 60h/Semestre, que envolve os conceitos do PMBOK, com foco em escopo, tempo e custo. Já quanto às disciplinas voltadas para o PDP, o curso de Engenharia de Produção conta com uma disciplina obrigatória denominada "Projeto de Produtos", com carga de 60h, desenvolvida durante 1 semestre. A ementa da disciplina engloba conceitos gerais de desenvolvimento de produto e de gerenciamento de projetos, sendo essa última voltada somente na área de conhecimento de escopo. Também nos cursos de Administração e Gestão da Informação, existe uma disciplina optativa de Desenvolvimento de Produtos, com carga de 30h, para apresentação dos principais conceitos sobre PDP e caracterização do envolvimento do gestor nesse processo. Entretanto, tal disciplina por ser optativa, não é oferecida semestralmente. 


\subsection{Análise}

Sobre a maturidade em PDP, em todas as onzes EJs estudadas observou-se que as empresas não percebem a necessidade de conhecimento em PDP, uma vez que as EJs entendem que os produtos oferecidos por elas possuem características de serviços, muitas vezes devido à intangibilidade. De uma maneira geral, observou-se também um foco na área de Marketing como sendo responsável pelo desenvolvimento do(s) produto(s) oferecido, sendo o planejamento de portfólio realizado com base nos dados levantados por áreas com esse foco. Porém, analisa-se dois pontos importantes: 1) nem todas as EJs estudadas oferecem serviços puros, como é o caso principalmente das EJs relacionadas com os cursos de engenharia; 2) muitos clientes atendidos desenvolvem produtos físicos e, muitas vezes, as EJs realizam propostas de melhoria em áreas que estão diretamente relacionadas com o PDP de seus clientes, assim seria importante o desenvolvimento de competências nessa disciplina, de forma a criar uma visão mais ampla sobre esse processo e oferecer um serviço mais robusto e eficiente aos clientes. A simples compreensão do PDP como um processo de negócio essencial por parte dos integrantes da EJs poderia modificar a visão estratégica da empresa.

Sobre a baixa maturidade em Gerenciamento de Projetos, em todas as EJs participantes foi possível observar que a falta dos conceitos básicos de GP tem como causa principal a ausência de uma disciplina na graduação sobre o tema. Assim, o aluno só passa a ter um primeiro contato com GP já dentro de EJ. Essa falta de conhecimento individual reflete-se também nas dimensões de equipe e organização. Observa-se a atribuição de uma maior importância para os eixos de escopo e tempo nas três dimensões.

Assim, o cenário observado de baixa maturidade nas competências de PDP e de GP mostra-se como um reflexo da falta de importância atribuída às duas disciplinas. O resultado deste estudo vai ao encontro do apontamento de Campos et al (2015), que mostra como necessidade prioritária para EJs o treinamento das competências de gestão estratégica.

Entretanto já existem fatores que podem influenciar positivamente no desenvolvimento das competências nas áreas estudadas. Uma vez que a competência de trabalho em equipe é bem desenvolvida, bem como a competência de comprometimento (MORETTO et al, 2004), observa-se a possibilidade de um impacto positivo nas três dimensões se a capacitação individual for otimizada. Como outro ponto positivo para o desenvolvimento da dimensão organização tem-se o tipo de estrutura organizacional utilizado pelas EJs, que é a estrutura matricial forte.

\section{CONCLUSÕES}

Este estudo estabeleceu um cenário das EJs relacionadas ao NEJ da UFU, considerando onze das vinte e uma EJs associadas. O cenário evidenciado é de baixa maturidade nas competências em gerenciamento de projetos nas dimensões do indivíduo, das equipes e da organização, bem como de um nível muito baixo em maturidade de PDP. Os resultados apresentados permitem concluir que existe uma falta de compreensão sobre a importância do PDP e de GP no desenvolvimento de uma organização, tal como uma EJ.

Considera-se também que o desenvolvimento de tais competências pode ser facilitado devido a outras competências mais fortemente trabalhadas nas EJs, tais como trabalho em equipe e comprometimento, bem como a estrutura organizacional já adotada pelas EJs.

Como propostas de melhorias para o desenvolvimento das competências de PDP e GP nas EJs, considera-se como medida mais adequada, primeiramente, o desenvolvimento da dimensão de competência individual, com o oferecimento de disciplinas na grade curricular voltadas tanto para área de gerenciamento de projetos como de desenvolvimento de produtos. 
Mas uma vez que a atualização curricular nos vários cursos pode demandar um trabalho burocrático independente das EJs, considera-se que o desenvolvimento de cursos de capacitação nas duas áreas seja uma alternativa viável, uma vez que dependeriam de parcerias entre as EJs e profissionais atuantes nas áreas, como docentes da própria UFU. Tais cursos podem contar com uma parceria com o NEJ, bem como com empresas da região, indo além do eixo de ensino, que está mais focado no desenvolvimento das habilidades específicas de cada curso de graduação, fortalecendo assim o eixo de extensão da universidade com a comunidade.

Considerando também que as EJs se encontram em ambientes inovadores, com desenvolvimento de novas ideias, sugere-se que, posteriormente a abordagem de gerenciamento ágil de projetos seja estudada como uma forma de potencializar seus projetos.

Este estudo possui limitações por ter tratado de uma única universidade, o que reflete um cenário local. Porém, dada a importância tanto dos estudos de PDP quanto de GP, aponta-se a possibilidade de estudos futuros para acompanhamento do desenvolvimento do tema, tanto na universidade estudada como em outras universidades que contam com empresas juniores.

\section{REFERÊNCIAS}

BRASIL JUNIOR. Conceito Nacional de Empresa Júnior, 2003. Disponível em: <https://www.brasiljunior.org.br/uploads/institutional/file/file/5/CNEJ.pdf>

BRASIL JUNIOR. Sancionada a lei das empresas juniores, 2016a. Disponível em: <https://www.brasiljunior.org.br/noticias/sancionada-a-lei-das-empresas-juniores>. Acesso em: 1 ago. 2017

BRASIL JUNIOR. Quão grandes seremos em 2017?, 2016b. Disponível em: <https://www.brasiljunior.org.br/brasil-junior/noticias/quao-grandes-seremos-em-2017>.

Acesso em: 1 ago. 2017

CAMPOS, E. B. D.; ABBAD, G. D. S.; MACEDO, A. G. A. P. S.; SILVA, N. P. Necessidades de Treinamento: Uma Proposta de Avaliação Estratégica no Contexto de Empresas Juniores Brasileiras. RAM. Revista de Administração Mackenzie, v. 16, n. 6, p. 126-158, 2015.

CLARK, K. B.; FUJIMOTO, T. Product development performance: strategy, organization, and management in the World Auto Industry. Boston: Harvard Business School Press, 1991.

DIAS, L. S.; OLIVEIRA, P. DE; MARCELINO, K. M. Modelo empresa júnior: Interação escola x empresa na formação gerencial do administrador e orientação ao empreendedor. Brazilian Journal of Development, v. 3, n. 1, p. 178-204, 2017.

GODOY, A. S. Pesquisa Qualitativa: tipos fundamentais. Revista de Administração de Empresas, v. 35, n. 3, p. 20-29, 1995.

GRABHER, G. The Project Ecology of Advertising: Tasks, Talents and Teams. Regional Studies, v. 36, n. 3, p. 245-262, maio 2002.

KRISHNAN, V.; ULRICH, K. T. Product Development Decisions: A Review of the Literature. Management Science, v. 47, n. 1, p. 1-21, 1 jan. 2001.

MATOS, F. DE. Empresa junior no brasil e no mundo. São Paulo: Martins Claret, 1997.

MORETTO NETO, L.; JUNKES, P. N.; ROSAURO, D. Z.; BENKO, F. Empresa Júnior Espaço de aprendizagem. Florianópolis: Pallotti, 2004. 
MUNDIM, A. P. F.; ROZENFELD, H.; AMARAL, D. C.; SILVA, S. L.; GUERRERO, V.; HORTA, L. C. Aplicando o cenário de desenvolvimento de produtos em um caso prático de capacitação profissional. Gestão \& Produção, v. 9, n. 1, p. 1-16, abr. 2002.

NEJ UFU. NEJ UFU. Disponível em: <https://www.facebook.com/NEJUFU/>. Acesso em: 1 ago. 2017.

PAHL G, BEITZ W, FELDHUSEN J, GROTE K-H. Engineering Design: A Systematic Approach. 3rd ed. Londres, UK: Spring-Verlag; 2007.

PATAH, L. A.; CARVALHO, M. M. DE. Alinhamento entre estrutura organizacional de projetos e estratégia de manufatura: uma análise comparativa de múltiplos casos. Gestão \& Produção, v. 16, n. 2, p. 301-312, 2009.

PMI. PMBOK: um guia do conhecimento em gerenciamento de projetos. 5a ed. Project Management Institute, Inc., 2013.

RABECHINI JUNIOR, R. et al. A organização da atividade de gerenciamento de projetos: os nexos com competências e estrutura. Gestão e Produção, v. 18, n. 2, p. 409-424, 2011.

ROZENFELD, H. et al. Gestão de desenvolvimento de produtos: uma referência para a melhoria do processo. São Paulo: Saraiva, 2006, 542 p.

ULRICH, K. T.; EPPINGER, S. D. Product Design and Development. 5th. ed. New York: Mcgraw-Hill Education, 2012.

VIEIRA, A. C. G. et al. Uma proposta para a nova estrutura organizacional da FEA Júnior USPAnais do VII SEMEAD. Anais...São Paulo: 2003.

WEISS, I. W.; WYSOCKI, R. K. 5-Phase project management. NewYork: Addison-Wesley, 1992. 